\title{
Real-Time Environmental Gamma Radiation Dose Rate Measurement around Major Nuclear and Radiological Facilities in Bangladesh
}

\author{
Sidratul Moontaha ${ }^{1}$, Dr. Mohammad Sohelur Rahman ${ }^{2}$, Dr. Md. Shafiqul Islamªnd Selina Yeasmin ${ }^{4}$ \\ ${ }^{1}$ MS Fellow, Department of Nuclear Engineering, University of Dhaka, Dhaka-1000, Bangladesh \\ ${ }^{2}$ Chief Scientific Officer, Health Physics Division, Atomic Energy Centre, 4 KaziNazrul Islam Avenue, \\ Shahbag, Dhaka-1000, Bangladesh \\ ${ }^{3}$ Associate Professor and Chairman, Department of Nuclear Engineering, University of Dhaka, Dhaka-1000, \\ Bangladesh \\ ${ }^{4}$ Chief Scientific Officer and Head, Health Physics Division, Atomic Energy Centre, 4 KaziNazrul Islam \\ Avenue,Shahbag, Dhaka-1000, Bangladesh
}

\begin{abstract}
:
Background: In this study, outdoor environmental gamma radiation dose rates were measured at area of Shahbag Thana under Dhaka city and Atomic Energy Research Establishment (AERE) Campus at Savar. Aim of the study: This kind of study is required to detect the presence of natural and artificial radionuclides (if any) releasing from nuclear and radiological facilities in the country or from neighbouring countries. Materials and Methods: The measurement was performed using a real-time portable radiation monitoring device from August-November 2017. The real-time portable radiation monitoring device was placed on tripod at 1 meter above the ground and data acquisition time for each monitoring point (MP) was 1 hour. Total 34 MP were selected around major nuclear and radiological facilities in Bangladesh for collection of dose rate due to gamma-ray. The MPs were marked-out using Global Positioning System (GPS) navigation. The GPS reading of the sampling locations were varied from E90 $23^{\prime} 40.08^{\prime \prime}$ to E90 $24^{\prime} 32.82^{\prime \prime}$ and from

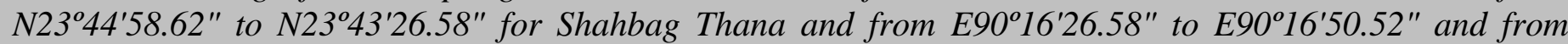
N2357'12.96" to N2357'6.12" for AERE Campus, Savar. Results: The measured dose rates due to natural radionuclides were ranged from $0.105 \pm 0.036056 \mu \mathrm{Sv} \cdot h^{-1}$ to $0.208065 \pm 0.106377 \mu \mathrm{Sv} . h^{-1}$ with an average of $0.141568 \pm 0.046995 \mu \mathrm{Sv} \cdot \mathrm{h}^{-1}$. The annual effective dose to the population from outdoor environmental gamma radiation was varied from $0.128772 \pm 0.044218 \mathrm{mSv}$ to $0.25517 \pm 0.130461 \mathrm{mSv}$ and the mean was found to be $0.17362 \pm 0.057635 \mathrm{mSv}$. This value is lower than some countries like India, China, Sweden, Italy and Czech Republic; and higher than Canada, Mexico, Indonesia, Korea, Turkey, Finland, Spain and some other countries. Conclusion: From this study, it was observed that there is no burden of population exposure due to man-made sources. Therefore, it can be concluded that adequate safety and radiation protection of nuclear \& radiological facilities had been ensured which is required for minimizing of unnecessary exposure to populations from man-made sources. The estimated mean annual effective dose found in this study is not expected to contribute significant additional hazard from the radiological health point of view.
\end{abstract}

Key words: Environmental radiation, Effective dose, In-situ, Gamma-Scout.

\section{Introduction}

From the beginning of life on earth, all living things have been exposed to radiation. Life started and developed in spite of, or possibly because of, radiation. It is disquieting to people that they coexist with radiation yet it cannot be seen, heard or felt ${ }^{[1]}$. One of the main external sources of ionizing radiation to the human body is represented by the gamma radiation emitted by naturally occurring radioisotopes. The most prominent naturally occurring radioisotopes are ${ }^{40} \mathrm{~K}$ and the radionuclides from the ${ }^{232} \mathrm{Th}$ and ${ }^{238} \mathrm{U}$ series with their decay products, 
which exist at trace levels in all ground formations. The majority of human exposure to ionizing radiation occurs from natural sources including cosmic rays and terrestrial radiation ${ }^{[2]}$. Exposure to terrestrial gamma radiation depends mostly on geographical characteristics of a place such as altitude, latitude and solar activity ${ }^{[3}$,

4]. Gamma ray accounts for the majority of external human exposures to radiation from all type of sources due to its high penetration ability ${ }^{[5]}$. Gamma radiation is ubiquitous. Great variations have been observed in environmental radiation levels and several international studies have been characterized gamma dose rates both in outdoor and indoor environments ${ }^{[6-14]}$.

Both laboratory and in-situ gamma spectroscopy are often used for monitoring and assessment of radioactivity and radiation dose rates in the environment due to both natural and anthropogenic sources ${ }^{[15-20]}$. In-situ techniques for measuring the activity concentration resulting from the gamma radiation and characterizing its sources with gamma ray spectrometer have been used successfully in outdoor and indoor environment ${ }^{[12 \text {, }}$ 21-23]

The presence of naturally occurring radionuclides in the environment may result in an external and internal dose received by a population exposed to them directly and through the ingestion and inhalation pathways. The assessment of the radiological impact on a population as a result of the radiation emitted by these radionuclides is important since they contribute to the collective dose of the population ${ }^{[24]}$. The aim of the present study is to measure outdoor environmntal gamma-ray dose rates from natural and artificial radionuclides (if any) releasing from nuclear facilities in the country or from neighbouring countries in normal operation or in case of incident/accident through in-situ technique.

Biological effects of ionizing radiation on human are evaluated based on the effective absorbed dose rate. Annual effective absorbed dose from background gamma radiation was determined using the equation (1), (2) and $(3)^{[25]}$.

$\mathrm{E}_{\text {total }}=\mathrm{E}_{\text {Out }}+\mathrm{E}_{\text {In }}=\left(\mathrm{D}_{\text {Out }} \times \mathrm{OF}_{\text {Out }}+\mathrm{D}_{\text {In }} \times \mathrm{OF}_{\text {In }}\right) \times \mathrm{T} \times \mathrm{CC}$

$\mathrm{E}_{\text {Out }}=\mathrm{T} \times \mathrm{D}_{\text {Out }} \times \mathrm{CC} \times \mathrm{O}_{\text {Fout }}(2)$

$\mathrm{E}_{\text {In }}=\mathrm{T} \times \mathrm{D}_{\text {In }} \times \mathrm{CC} \times \mathrm{OF}_{\text {In }}(3)$

Where, $\mathrm{E}_{\text {total }}=$ Total annual effective absorbed dose rate $(\mathrm{mSv} / \mathrm{y}) ; \mathrm{E}_{\mathrm{In}}=$ Indoor annual effective absorbed dose rate $(\mathrm{mSv} / \mathrm{y}) ; \mathrm{E}_{\text {Out }}=$ Outdoor annual effective absorbed dose rate $(\mathrm{mSv} / \mathrm{y}) ; \mathrm{T}=$ Time in hours $(8760$ hours for a year $) ; \mathrm{D}_{\text {In }}=$ Absorbed dose rate in indoor $(\mathrm{nSv} / \mathrm{h}) ; \mathrm{D}_{\text {Out }}=$ Absorbed dose rate in outdoor $(\mathrm{nSv} / \mathrm{h}) ; \mathrm{OF}_{\text {In }}=\mathrm{Indoor}$ occupancy factor $(80 \%$ for indoor $) ; \mathrm{OF}_{\text {out }}=$ Outdoor occupancy factors $(20 \%$ for outdoor $)$; And $\mathrm{CC}=$ Conversion coefficient $\left(0.7 \mathrm{~Sv}^{\bullet} \mathrm{Gy}^{-1}\right.$ for adult); Reported by UNSCEAR to convert absorbed dose in air to the effective dose in human ${ }^{[4]}$.

\section{Materials and Methods}

\subsection{In-Situ gamma-ray dose rate measurement}

Real-time gamma-ray dose rate measurement was performed around major nuclear and radiological facilities in Bangladesh through In-Situ technique using a portable radiation monitoring device (GAMMA SCOUT).The Gamma-Scout is a general purpose Geiger counter that measures alpha, beta, gamma and x-ray radiation. It has proven to be useful in the medical, nuclear, mining, metal scrap and foundry industries. It is also used by first responders, police, customs and border control, hobbyists, rock hounds and in personal or home survival kits.The Gamma-Scout is German designed and manufactured, built with a solid Novadur exterior. The Gamma-Scout Geiger counter has a large digital display that can display the radiation detected as dose rate $(\mu \mathrm{Sv} / \mathrm{hr})$ or in pulses (total count or per second). There is also an analog logarithmic bar chart to quickly visualize the magnitude of the measured dose rate. The unit has a battery indicator, multiple unit conversion, real-time dose rate and cumulative dose display functions and programmable logging and alert functions. Advanced functions include PC data download via USB cable and an ultra-low current power circuit for extended battery life ${ }^{[26]}$.

\subsection{Gamma-ray calibration sources}


The GAMMA SCOUT was calibrated using standard sources such as ${ }^{133} \mathrm{Ba}$, ${ }^{137} \mathrm{Cs}$ and ${ }^{60} \mathrm{Co}$ during manufacturing time. The GAMMA SCOUT was calibrated from Secondary Standard Dosimetry Laboratory under Bangladesh Atomic Energy Commission. The tested device must be within a confidence interval of 5\% against a master, which in turn, is adjusted to a gauged reference ${ }^{137} \mathrm{Cs}$ emitter ${ }^{[27]}$.Under environmental radiation, the counter tube is not subject to fatigue and, therefore, will not require re-calibration. However, if the user holds ISO certification, periodical calibration is mandatory ${ }^{[26]}$.

\subsection{The Site}

The study had been performed over two sites; one is Shahbag Thana under Dhaka city and another is Atomic Energy Research Establishment (AERE) in Savar where major nuclear and radiological facilities were established. These sites are located from E90 23'40.08" to E90 24'32.82" and from N23 44'58.62" to

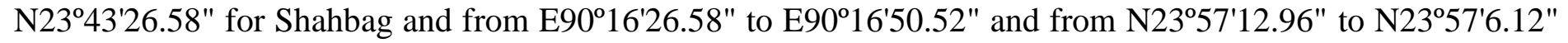
for AERE, Savar. Total thirty four locations (twenty seven in Shahbag Thana and seven in AERE had been monitored to measure outdoor environmental gamma radiation dose rate. These gamma dose rates had been measured for one hour in each location using portable Gamma-Scout detector through in-situ technique from August to November 2017. The field measurement of environmental gamma radiation was based on the assumption that there exist laterally uniform distribution of natural radionuclides in the environment and that the vertical contribution from the soil is limited to the first horizon $(10 \mathrm{~cm}$ to $30 \mathrm{~cm})$. The detector was set on a tripod at $1 \mathrm{~m}$ height from the ground level. On the other hand, the latitude and altitude of the site locations had been measured by Global Positioning System (GPS) navigation. Figure 1 indicates all the monitoring points in Shahbag Thana under Dhaka city from where the outdoor environmental gamma dose rates were measured. 


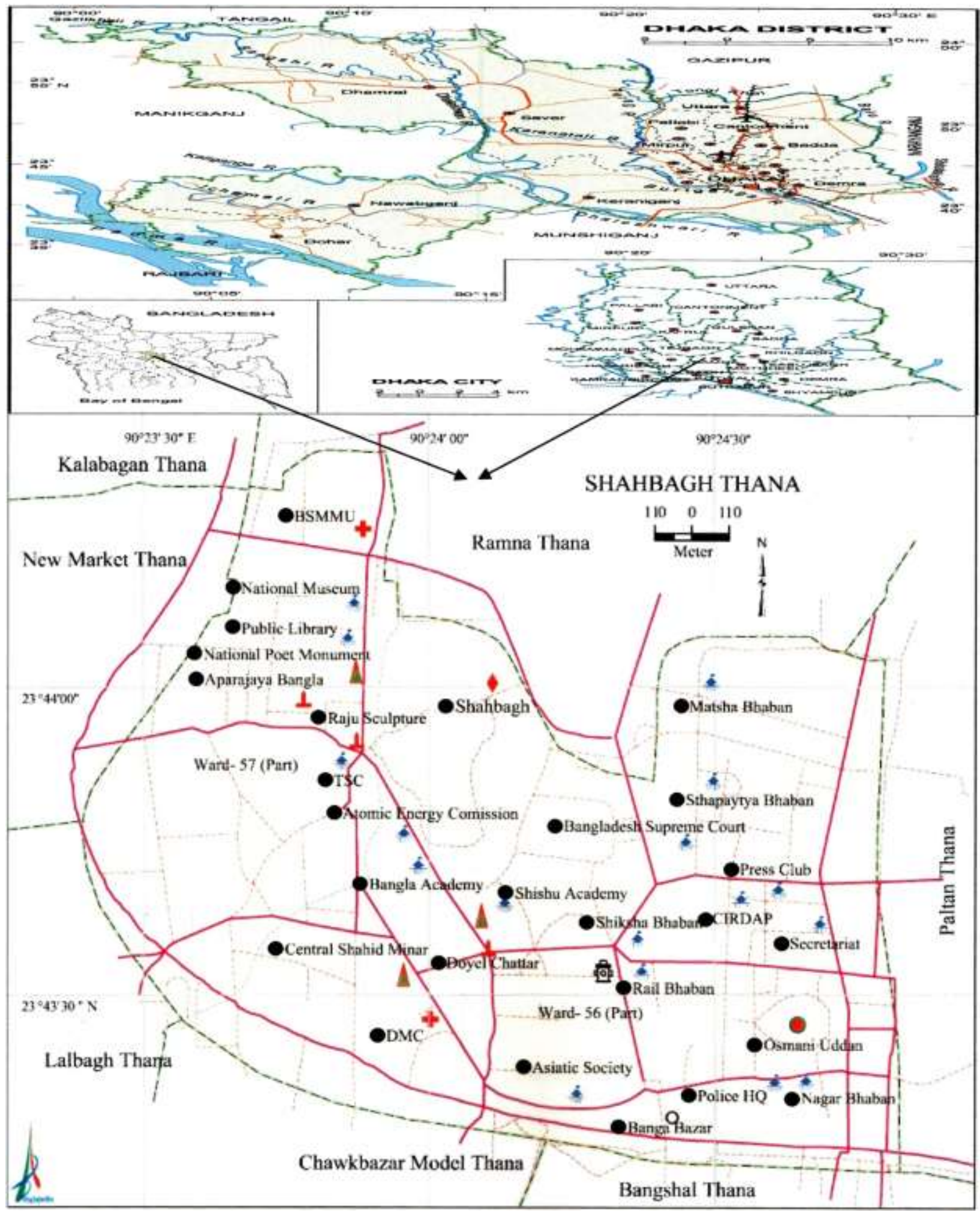

Figure 1: Shows the location $(\bullet)$ in Shahbag Thana under Dhaka city where outdoor environmental gamma radiation measurement was performed using portable Gamma-Scout detector through in-situ technique ${ }^{[27]}$.

\section{Results and Discussion}

\subsection{Absorbed dose rate and annual effective dose}

The average gamma radiation dose rate of thirty four monitoring points was found to be $0.141568 \pm 0.046995 \mu \mathrm{Sv} / \mathrm{hr}$; the minimum dose rate was found to be $0.105 \pm 0.036056 \mu \mathrm{Sv} / \mathrm{hr}$ and the maximum 
dose rate was found to be $0.208065 \pm 0.106377 \mu \mathrm{Sv} / \mathrm{hr}$. So the range of the outdoor environmental gamma radiation dose rate in this whole study area was varied from $0.105 \pm 0.036056 \mu \mathrm{Sv} / \mathrm{hr}$ to $0.208065 \pm 0.106377 \mu \mathrm{Sv} / \mathrm{hr}$ with an average of $0.141568 \pm 0.046995 \mu \mathrm{Sv} / \mathrm{hr}$. Using the conversion factor of 0.7 Sv/Gy as recommended by UNSCEAR $2000^{[28]}$ and considering that people in Bangladesh spend approximately $20 \%$ of their time outdoor and remaining $80 \%$ of time indoor; the outdoor annual effective gamma radiation dose received by people in Shahbag Thana and AERE Campus has been calculated and the calculated data is given in Table 1. From the table, the average annual effective dose was found to be $0.17362 \pm$ $0.057635 \mathrm{mSv}$. The minimum annual effective dose was found to be $0.128772 \pm 0.044218 \mathrm{mSv}$ and the maximum was $0.25517 \pm 0.130461 \mathrm{mSv}$. So the outdoor environmental annual effective gamma radiation dose for the whole study area is ranged from $0.128772 \pm 0.044218 \mathrm{mSv}$ to $0.25517 \pm 0.130461 \mathrm{mSv}$ with an average of $0.17362 \pm 0.057635 \mathrm{mSv}$.

Table 1: Outdoor environmental gamma dose rate and annual effective dose for 34 MPs at Shahbag Thana and AERE Campus at Savar

\begin{tabular}{|c|c|c|c|c|c|}
\hline $\begin{array}{l}\text { Monitorin } \\
\text { g point }\end{array}$ & Name of Place & $\begin{array}{l}\text { Latitude/Altitud } \\
\text { e }\end{array}$ & $\begin{array}{l}\text { Gamma } \\
\text { Dose Rate } \\
\text { Range } \\
(\mu \text { Sv/hr })\end{array}$ & $\begin{array}{l}\text { Mean Gamma } \\
\text { Dose Rate with SD } \\
(\mu \mathrm{Sv} / \mathrm{hr})\end{array}$ & $\begin{array}{l}\begin{array}{l}\text { Annual } \\
\text { Effective }\end{array} \begin{array}{lr}\text { Mean } \\
\text { Dose with } \\
(\mathrm{mSv})\end{array}\end{array}$ \\
\hline 1 & BSMMU & $\begin{array}{l}\text { N2344'22.56" } \\
\text { E90'23'44.64" }\end{array}$ & $\begin{array}{l}(0.05- \\
0.32)\end{array}$ & $0.185 \pm 0.08226$ & $0.226884 \pm 0.100883$ \\
\hline 2 & Shahbag & $\begin{array}{l}\text { N234'16.14" } \\
\text { E90²3'44.7" }\end{array}$ & $\begin{array}{l}(0.07- \\
0.18)\end{array}$ & $0.125 \pm 0.036056$ & $0.1533 \pm 0.044218$ \\
\hline 3 & National Museum & $\begin{array}{l}\text { N2344'14.46" } \\
\text { E90²3'43.44" }\end{array}$ & $\begin{array}{l}(0.07- \\
0.22)\end{array}$ & $0.145 \pm 0.04761$ & $0.177828 \pm 0.058388$ \\
\hline 4 & Public Library & $\begin{array}{l}\text { N234'11.28" } \\
\text { E90'23'40.08" }\end{array}$ & $\begin{array}{l}(0.05- \\
0.45)\end{array}$ & $\begin{array}{l}0.2080645 \pm 0.10637 \\
7\end{array}$ & $\begin{array}{l}0.2551703 \pm 0.13046 \\
1\end{array}$ \\
\hline 5 & $\begin{array}{ll}\text { National } & \text { Poet } \\
\text { Monument } & \\
\end{array}$ & $\begin{array}{l}\text { N234'ㄴ.12" } \\
\text { E90 } 23^{\prime} 43.38^{\prime \prime}\end{array}$ & $\begin{array}{l}(0.05- \\
0.20)\end{array}$ & $0.125 \pm 0.04761$ & $0.1533 \pm 0.058388$ \\
\hline 6 & Aparajaya Bangla & $\begin{array}{l}\text { N2344'58.62" } \\
\text { E90²3'43.74" }\end{array}$ & $\begin{array}{l}(0.05- \\
0.17)\end{array}$ & $0.11 \pm 0.038944$ & $0.134904 \pm 0.047761$ \\
\hline 7 & Raju Sculpture & $\begin{array}{l}\text { N2343'56.16" } \\
\text { E90²3'42.6" }\end{array}$ & $\begin{array}{l}(0.07- \\
0.24)\end{array}$ & $0.155 \pm 0.053385$ & $0.190092 \pm 0.065472$ \\
\hline 8 & TSC & $\begin{array}{l}\text { N2343'54.96" } \\
\text { E90 } 23^{\prime} 45.96^{\prime \prime}\end{array}$ & $\begin{array}{l}(0.05- \\
0.25)\end{array}$ & $0.15 \pm 0.062048$ & $0.18396 \pm 0.076096$ \\
\hline 9 & $\begin{array}{l}\text { Atomic Energy } \\
\text { Commission }\end{array}$ & $\begin{array}{l}\mathrm{N} 23^{\circ} 43^{\prime} 52.68^{\prime \prime} \\
\mathrm{E} 90^{\circ} 23^{\prime} 49.62^{\prime \prime}\end{array}$ & $\begin{array}{l}(0.07- \\
0.20)\end{array}$ & $0.135 \pm 0.041833$ & $0.165564 \pm 0.051304$ \\
\hline 10 & Bangla Academy & $\begin{array}{l}\text { N2343'49.08" } \\
\text { E90²3'52.92" }\end{array}$ & $\begin{array}{l}(0.07- \\
0.19)\end{array}$ & $0.13 \pm 0.038944$ & $0.159432 \pm 0.047761$ \\
\hline 11 & DoyelChattar & $\begin{array}{l}\text { N2343'41.4" } \\
\text { E90²4'0.12" }\end{array}$ & $\begin{array}{l}(0.07- \\
0.17)\end{array}$ & $0.12 \pm 0.033166$ & $0.147168 \pm 0.040675$ \\
\hline 12 & Shishu Academy & $\begin{array}{l}\mathrm{N} 23^{\circ} 43^{\prime} 41.58^{\prime \prime} \\
\mathrm{E} 90^{\circ} 24 ' 4.2^{\prime \prime}\end{array}$ & $\begin{array}{l}(0.10- \\
0.25)\end{array}$ & $0.175 \pm 0.04761$ & $0.21462 \pm 0.058388$ \\
\hline 13 & $\begin{array}{l}\text { Bangladesh } \\
\text { Supreme Court }\end{array}$ & $\begin{array}{l}\text { N234'42.54" } \\
\text { E90²4'13.74" }\end{array}$ & $\begin{array}{l}(0.08- \\
0.20)\end{array}$ & $0.14 \pm 0.038944$ & $0.171696 \pm 0.047761$ \\
\hline 14 & SikhshaBhaban & $\begin{array}{l}\text { N2343'45.18" } \\
\text { E90'24'17.22" }\end{array}$ & $\begin{array}{l}(0.07- \\
0.19)\end{array}$ & $0.13 \pm 0038944$ & $0.159432 \pm 0.047761$ \\
\hline 15 & CIRDAP & $\begin{array}{l}\text { N2343'46.32" } \\
\text { E90²4'18.3" }\end{array}$ & $\begin{array}{l}(0.07- \\
0.22)\end{array}$ & $0.14 \pm 0.050498$ & $0.171696 \pm 0.06193$ \\
\hline 16 & Secretariat & $\begin{array}{l}\text { N2343'43.02" } \\
\text { E90²4'25.92" }\end{array}$ & $\begin{array}{l}(0.07- \\
0.23)\end{array}$ & $0.15 \pm 0.050498$ & $0.18396 \pm 0.06193$ \\
\hline
\end{tabular}




\begin{tabular}{|c|c|c|c|c|c|}
\hline 17 & Press Club & $\begin{array}{l}\text { N23 } 3^{\circ} 43^{\prime} 47.16^{\prime \prime} \\
\text { E90 } 24^{\circ} 26.64^{\prime \prime}\end{array}$ & $\begin{array}{l}0.04- \\
0.18)\end{array}$ & $0.11 \pm 0.044721$ & $0.134904 \pm 0.054846$ \\
\hline 18 & Rail Bhaban & $\begin{array}{l}\text { N23연 } 40.2^{\prime \prime} \\
\text { E90 } 24^{\prime} 21.48^{\prime \prime}\end{array}$ & $\begin{array}{l}(0.07- \\
0.17)\end{array}$ & $0.12 \pm 0.033166$ & $0.147168 \pm 0.040675$ \\
\hline 19 & OsmaniUddan & $\begin{array}{l}\text { N23ㄴ3'39.18" } \\
\text { E90'24'32.82" }\end{array}$ & $\begin{array}{l}(0.07- \\
0.21)\end{array}$ & $0.14 \pm 0.044721$ & $0.171696 \pm 0.054846$ \\
\hline 20 & Police HQ & $\begin{array}{l}\text { N2343'29.16"; } \\
\text { E90'24'26.28" }\end{array}$ & $\begin{array}{l}(0.07- \\
0.20)\end{array}$ & $0.135 \pm 0.041833$ & $0.165564 \pm 0.051304$ \\
\hline 21 & Nagar Bhaban & $\begin{array}{l}\text { N234'ㄹ'28.74"; } \\
\text { E90'24'31.08" }\end{array}$ & $\begin{array}{l}(0.05- \\
0.16)\end{array}$ & $0.105 \pm 0.036056$ & $0.128772 \pm 0.044218$ \\
\hline 22 & MatshaBhaban & $\begin{array}{l}\text { N234' } 1.98^{\prime \prime} ; \\
\text { E90'24'14.28" }\end{array}$ & $\begin{array}{l}(0.07- \\
0.19)\end{array}$ & $0.13 \pm 0.038944$ & $0.159432 \pm 0.047761$ \\
\hline 23 & $\begin{array}{l}\text { SthapaytyaBhaba } \\
\mathrm{n}\end{array}$ & $\begin{array}{l}\text { N234ㄴ'57"; } \\
\text { E90 } 24^{\circ} 16.62^{\prime \prime}\end{array}$ & $\begin{array}{l}(0.08- \\
0.27)\end{array}$ & $0.175 \pm 0.059161$ & $0.21462 \pm 0.072555$ \\
\hline 24 & $\begin{array}{l}\text { Central } \\
\text { ShaheedMinar }\end{array}$ & $\begin{array}{l}\text { N234'ㄱ' } 39.96^{\prime \prime} \\
\text { E90 } 23^{\prime} 49.32^{\prime \prime}\end{array}$ & $\begin{array}{l}(0.10- \\
0.21)\end{array}$ & $0.155 \pm 0.036056$ & $0.190092 \pm 0.044218$ \\
\hline 25 & DMC & 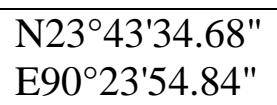 & $\begin{array}{l}(0.07- \\
0.20)\end{array}$ & $0.135 \pm 0.041833$ & $0.165564 \pm 0.051304$ \\
\hline 26 & Bangabzar & 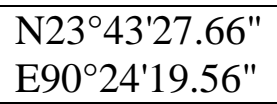 & $\begin{array}{l}(0.04- \\
0.19)\end{array}$ & $0.115 \pm 0.04761$ & $0.141036 \pm 0.058388$ \\
\hline 27 & Asiatic Society & 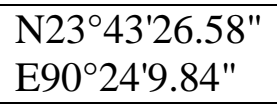 & $\begin{array}{l}(0.02- \\
0.23)\end{array}$ & $0.15 \pm 0.057271$ & $0.18396 \pm 0.070238$ \\
\hline 28 & $\begin{array}{ll}\text { Nuclear } & \text { Medical } \\
\text { Physics } & \text { Institute } \\
\text { (NMPI) } & \\
\end{array}$ & $\begin{array}{l}\mathrm{N} 23^{\circ} 57^{\prime} 12.9^{\prime \prime} \\
\mathrm{E} 90^{\circ} 16^{\prime} 26.58^{\prime \prime}\end{array}$ & $\begin{array}{l}(0.07- \\
0.20)\end{array}$ & $0.135 \pm 0.041833$ & $0.165564 \pm 0.051304$ \\
\hline 29 & $\begin{array}{lr}\text { Centre for } \\
\text { Research Reactor } \\
\text { (CRR) }\end{array}$ & $\begin{array}{l}\text { N235'10.08" } \\
\text { E90 } 16^{\circ} 45.78^{\prime \prime}\end{array}$ & $\begin{array}{l}(0.10- \\
0.24)\end{array}$ & $0.17 \pm 0.044721$ & $0.208488 \pm 0.054846$ \\
\hline 30 & $\begin{array}{l}\text { Radioactive } \\
\text { Waste } \\
\text { management } \\
\text { facility (INST) }\end{array}$ & $\begin{array}{l}\text { N235'1‥46" } \\
\text { E90'16'50.52" }\end{array}$ & $\begin{array}{l}(0.10- \\
0.18)\end{array}$ & $0.14 \pm 0.027386$ & $0.171696 \pm 0.033586$ \\
\hline 31 & $\begin{array}{l}\text { Radioisotope } \\
\text { production } \\
\text { facility (INST) }\end{array}$ & $\begin{array}{l}\mathrm{N} 23^{\circ} 57^{\prime} 10.98^{\prime \prime} \\
\mathrm{E} 90^{\circ} 16^{\prime} 48.48^{\prime \prime}\end{array}$ & $\begin{array}{l}(0.05- \\
0.18)\end{array}$ & $0.115 \pm 0.041833$ & $0.141036 \pm 0.051304$ \\
\hline 32 & $\begin{array}{l}\text { Tandem } \\
\text { Accelerator } \\
\text { facility }\end{array}$ & $\begin{array}{l}\mathrm{N} 23^{\circ} 57^{\prime} 12.96^{\prime \prime} \\
\mathrm{E} 90^{\circ} 16^{\prime} 47.04^{\prime \prime}\end{array}$ & $\begin{array}{l}(0.07- \\
0.23)\end{array}$ & $0.15 \pm 0.050498$ & $0.18396 \pm 0.06193$ \\
\hline 33 & $\begin{array}{lr}\text { Institute } & \text { of } \\
\text { radiation } & \text { and } \\
\text { polymer } & \\
\text { technology } & \\
\text { (IRPT) } & \end{array}$ & $\begin{array}{l}\text { N235'․․12" } \\
\text { E90'16'38.82" }\end{array}$ & $\begin{array}{l}(0.07- \\
0.16)\end{array}$ & $0.115 \pm 0.030277$ & $0.141036 \pm 0.037131$ \\
\hline 34 & $\begin{array}{l}\text { Institute of Food } \\
\text { and Radiation } \\
\text { Biology (IFRB) }\end{array}$ & $\begin{array}{l}\text { N235'․․54" } \\
\text { E90'16'41.28" }\end{array}$ & $\begin{array}{l}(0.10- \\
0.32)\end{array}$ & $0.195263 \pm 0.065181$ & $\begin{array}{l}0.2394707 \pm 0.07993 \\
8\end{array}$ \\
\hline
\end{tabular}

From Table 1, it is stated that the minimum outdoor environmental gamma radiation dose rate was found in Nagar Bhaban and the measured value was $0.105 \pm 0.036056 \mu \mathrm{Sv} / \mathrm{hr}$. On the other hand, the maximum outdoor 
environmental gamma radiation dose rate was found in Public Library and the measured value was $0.208065 \pm 0.106377 \mu \mathrm{Sv} / \mathrm{hr}$. A wide variation is shown in measurements of gamma dose which is varied from $0.128772 \pm 0.044218 \mathrm{mSv}$ to $0.25517 \pm 0.130461 \mathrm{mSv}$ because of the geographical structures, positions and the variation in latitude and altitude of the study area.

It is also indicated that there is a direct relationship between background dose rate and latitude of the region. The minimum annual effective dose was found in Nagar Bhaban, located in south eastern part of Shahbag Thana. This place is located in lower latitude region and has lower background gamma radiation in comparison to higher latitude regions of Shahbag Thana. One main reason for this phenomenon is magnetic field of the earth which increases by latitude and reach to the optimum value at poles. Magnetic field of the earth can affect slow moving charged particles and diverts them towards the poles ${ }^{[29,30]}$.

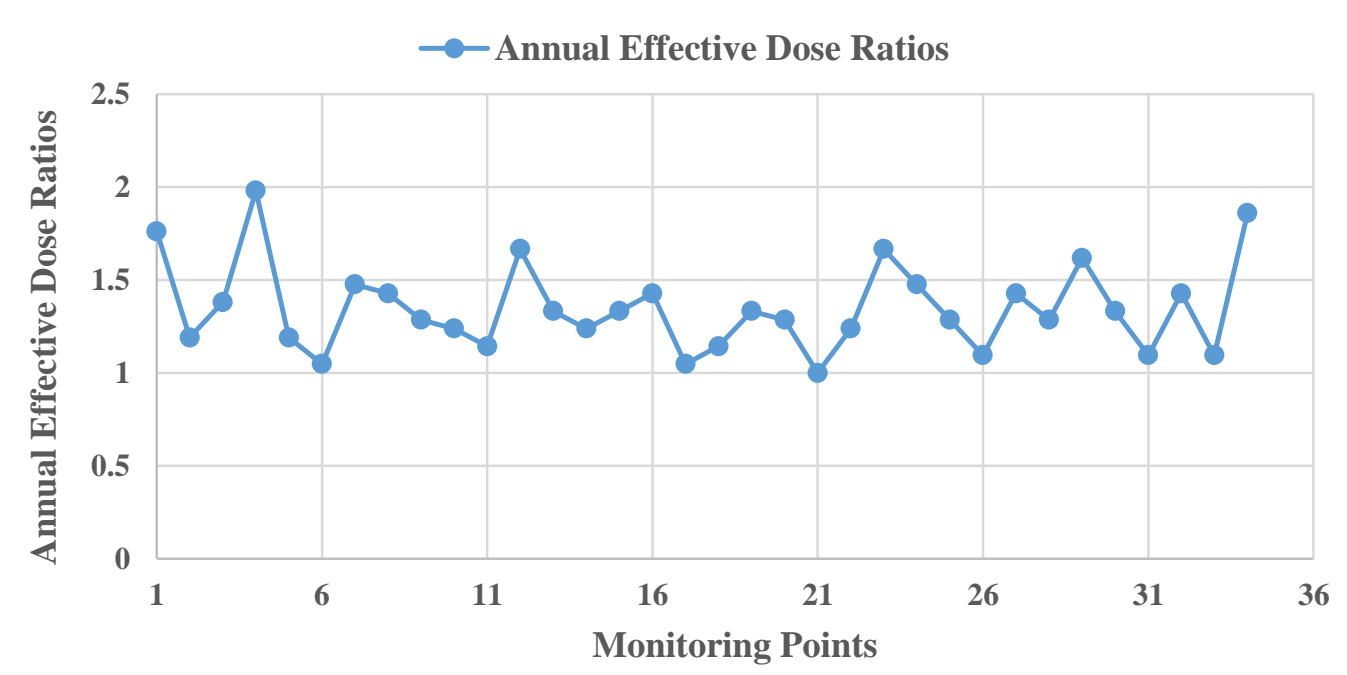

Figure 2: Outdoor annual effective dose values normalized to the minimum annual effective dose for each monitoring point.

The frequency distribution of the environmental gamma dose rates follow a normal type distribution as shown in Figure 3.

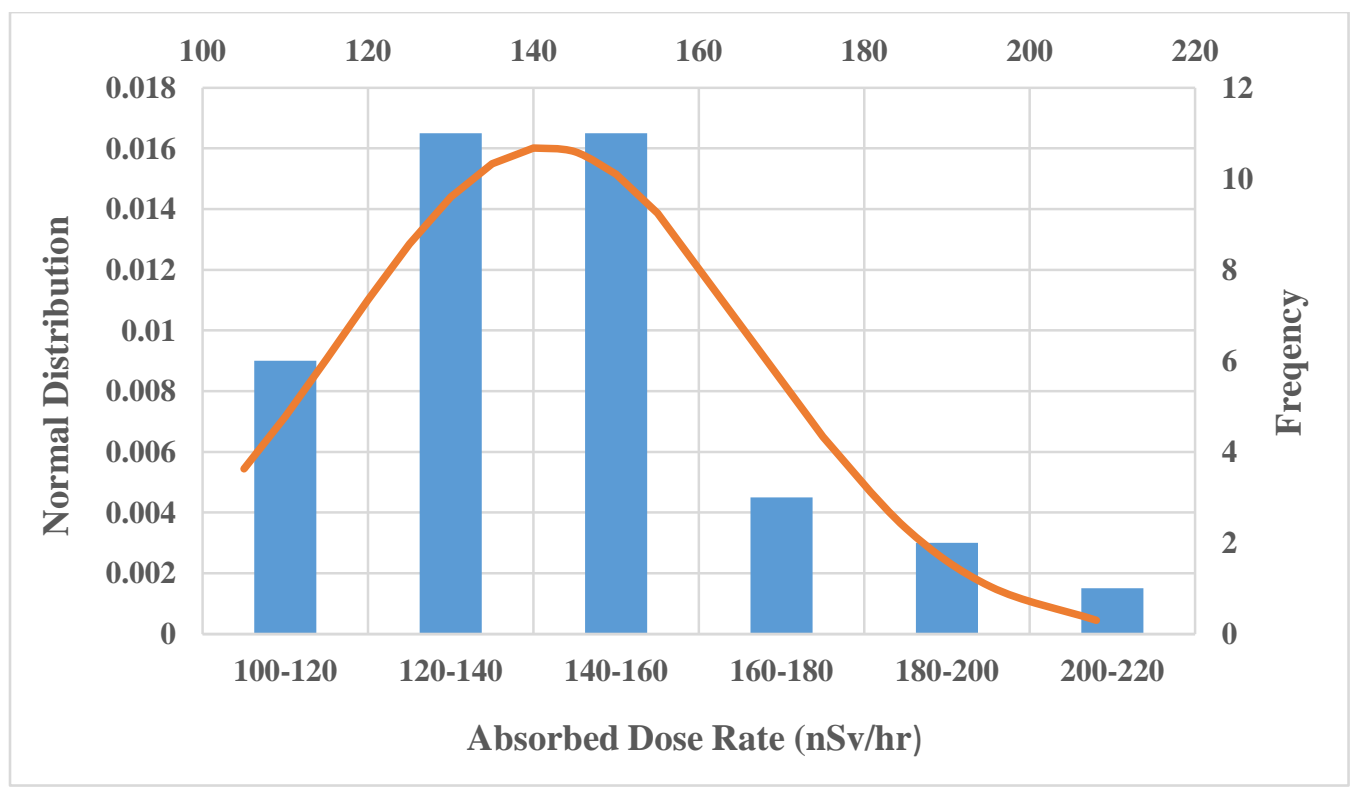


Figure 3: Frequency distribution of the absorbed dose rates (nSv/hr) at monitoring area follow normal distribution.

\subsection{Comparison with Other Countries}

From Table 2, it is shown that the maximum range of dose rate and annual effective dose, observed in this study, is lower than India, China, Sweden, Italy and Czech Republic; and higher than Canada, Mexico, Indonesia, Korea, Turkey, Finland, Spain and some other countries.

Table 2: Outdoor Environmental Gamma radiation dose rate range and annual effective dose range of Shahbag

Thana and AERE Campus, Savar and comparison with other countries [UNSCEAR 2008] ${ }^{[31]}$

\begin{tabular}{|c|c|c|c|c|c|}
\hline SL No. & Name of Country & $\begin{array}{l}\text { Range of Dose } \\
\text { Rate }(\mu \mathrm{Sv} / \mathrm{hr})\end{array}$ & $\begin{array}{l}\text { Mean } \\
\text { Dose } \\
\text { Rate } \\
(\mu \mathrm{Sv} / \mathrm{hr})\end{array}$ & $\begin{array}{lr}\text { Range of } & \text { Annual } \\
\text { Effective } & \text { Dose } \\
(\mathrm{mSv}) & \end{array}$ & $\begin{array}{l}\text { Mean } \\
\text { Annual } \\
\text { Effective } \\
\text { Dose } \\
(\mathbf{m S v}) \\
\end{array}$ \\
\hline 1. & $\begin{array}{ll}\text { Libyan } & \text { Arab } \\
\text { Jamahiriya } & \end{array}$ & $0.048-0.054$ & 0.051 & $0.058867-0.066226$ & 0.062546 \\
\hline 2. & Mauritius & $0.08-0.126$ & 0.098 & $0.098112-0.154526$ & 0.120187 \\
\hline 3. & $\begin{array}{l}\text { Tanzania (United } \\
\text { Rep. of) }\end{array}$ & $0.098-0.121$ & 0.104 & $0.120187-0.148394$ & 0.127546 \\
\hline 4. & Canada & $0.031-0.075$ & 0.054 & $0.038018-0.09198$ & 0.066226 \\
\hline 5. & Mexico & $0.023-0.184$ & 0.0883 & $0.028207-0.225658$ & 0.108291 \\
\hline 6. & Costa Rica & $0.035-0.147$ & 0.0659 & $0.042924-0.180281$ & 0.08082 \\
\hline 7. & Cuba & $0.038-0.196$ & 0.055 & $0.046603-0.240374$ & 0.067452 \\
\hline 8. & Azerbaijan & $0.075-0.205$ & 0.14 & $0.09198-0.251412$ & 0.171696 \\
\hline 9. & China & $0.0116-0.523$ & 0.0815 & $0.014226-0.641407$ & 0.099952 \\
\hline 10. & India $^{[32]}$ & $0.251-0.879$ & 0.4494 & $0.307826-1.078006$ & 0.551144 \\
\hline 11. & Indonesia & $0.045-0.102$ & 0.0675 & $0.055188-0.125093$ & 0.082782 \\
\hline 12. & Korea & $0.018-0.2$ & 0.079 & $0.022075-0.24528$ & 0.096886 \\
\hline 13. & $\begin{array}{l}\text { Islamic Republic } \\
\text { of Iran }\end{array}$ & $0.069-0.1876$ & 0.1115 & $0.084622-0.230073$ & 0.136744 \\
\hline 14. & Turkey & $0.032-0.094$ & 0.065 & $0.039245-0.115282$ & 0.079716 \\
\hline 15. & Denmark & $0.056-0.101$ & 0.066 & $0.068678-0.123866$ & 0.080942 \\
\hline 16. & Finland & $0.077-0.171$ & 0.103 & $0.094433-0.209714$ & 0.126319 \\
\hline 17. & Lithuania & $0.079-0.115$ & 0.095 & $0.096886-0.141036$ & 0.116508 \\
\hline 18. & Sweden & $0.04-0.63$ & 0.097 & $0.049056-0.772632$ & 0.118961 \\
\hline 19. & Belgium & $0.045-0.12$ & 0.076 & $0.055188-0.147168$ & 0.093206 \\
\hline 20. & Ireland & $0.035-0.143$ & 0.065 & $0.042924-0.175375$ & 0.079716 \\
\hline 21. & Italy & $0.057-0.243$ & 0.112 & $0.069905-0.298015$ & 0.137357 \\
\hline 22. & Spain & $0.05-0.129$ & 0.085 & $0.06132-0.158206$ & 0.104244 \\
\hline 23. & Switzerland & $0.053-0.155$ & 0.081 & $0.064999-0.190092$ & 0.099338 \\
\hline 24. & Bulgaria & $0.075-0.14$ & 0.1 & $0.09198-0.171696$ & 0.12264 \\
\hline 25. & Czech Republic & $0.04-0.285$ & 0.1 & $0.049056-0.349524$ & 0.12264 \\
\hline 26. & Poland & $0.051-0.1262$ & 0.0809 & $0.062546-0.154772$ & 0.099216 \\
\hline 27. & Romania & $0.052-0.163$ & 0.092 & $0.063773-0.199903$ & 0.112829 \\
\hline 28. & Albania & $0.0772-0.103$ & 0.094 & $0.094678-0.126319$ & 0.115282 \\
\hline 29. & Croatia & $0.077-0.14$ & 0.115 & $0.094433-0.171696$ & 0.141036 \\
\hline 30. & New Zealand & $0.034-0.122$ & 0.076 & $0.041698-0.149621$ & 0.093206 \\
\hline
\end{tabular}


In this study, the estimated mean annual effective dose is $0.17362 \mathrm{mSv}$ which is not expected to contribute significant additional hazard from the radiological health point of view. Due to comparison purposes, the annual dose limit for members of the public according to ICRP 103 (2007 recommendation) is $1 \mathrm{mSv} / \mathrm{year}{ }^{\text {[33] }}$. This limit is applicable to practices giving rise to controllable exposure and is not applicable to doses received from natural sources.

\section{Conclusion}

The present study has measured the outdoor environmental gamma radiation dose rates at the area of Shahbag Thana under Dhaka city and Atomic Energy Research Establishment (AERE) Campus, Savar where major nuclear and radiological facilities were established of Bangladesh. The estimated mean annual effective dose of $0.17362 \mathrm{mSv}$ is not expected contribute a statistically significant additional hazard from the radiological health point of view. For comparison purposes, the annual dose limit for members of the public as per ICRP (2011 recommendation) is $1 \mathrm{mSv} \cdot \mathrm{y}^{-1}$. This limit is applicable to practices giving rise to controllable exposure and is not applicable to doses received from natural sources. This type of study is very important for Bangladesh because the usage of radioactive material is increasing day by day in the various fields like medicine, industry and research \& education. Moreover, real-time environmental gamma radiation monitoring is crucial to generate the baseline database from natural sources and releasing (if any) from nuclear installations in the country or from neighboring countries in normal operations or in case of accident/incident. This kind of study will also be needed for measurement of environmental radioactivity in and around the Rooppur Nuclear Power Project (RNPP) site area. From this study, it can be concluded that the assessment of the dose level of the area did not detect the presence of any artificial radionuclides and thus no significant impact of the extensive usage of radioactive materials in and around Shahbag Thana and AERE Campus and no radiation burden to the environment. Finally, it can be concluded that adequate safety and radiation protection of nuclear \& radiological facilities had been ensured which is required for minimizing of unnecessary exposure to populations from manmade sources.

\section{Acknowledgement}

The authors would like to thanks relevant personnel of Health Physics Division, Atomic Energy Centre Dhaka for their support.

\section{References}

[1] ThormodHenriksen and Biophysics group at UiO, "Radiation and Health", Updated 2013.

[2] M. Charles, UNSCEAR Report, "Sources and Effects of Ionizing Radiation," J. Radiol. Prot., 21, pp.83-86, 2000.

[3] Agency for Toxic Substances and Disease Registry (ATSDR): Toxicological profile for ionizing radiation. Atlanta, GA: US, Department of Health and Human Services, Public Health Service, 1999.

[4] UNSCEAR Report, "Sources and Effects of Ionizing Radiation," Annex A: Dose assessment methodologies, New York, United Nations Scientific Committee on the effects of atomic radiation, Vol.1, 2000.

[5] F.S. Al-Saleh, "Measurement of indoor gamma radiation and radon concentrations in dwellings of Riyadh City, Saudi Arabia," ApplRadiatIsot, 65, pp. 843-848, 2007.

[6] F.H. Al-Ghorable, "Measurement of environmental terrestrial gamma radiation dose rate in three mountainous locations in the western region of Saudi Arabia," Environ Res, 98, pp. 160-166, 2005.

[7] H. Arvela, "Population distribution of doses from natural radiation in Finland," IntCongrSer, 1225, pp. 9-14, 2002.

[8] L. Rybach, D. Bachler, B. Bucher, G. Schwarz, "Radiation doses of Swiss population from external sources,"J Environ Radiat, 62, pp. 277-286, 2002. 
[9] F. Sagnatchi, M. Salouti, A. Eslami, "Assessment of annual effective dose due to natural gamma radiation in Zanjan (Iran)," RadiatProtDosim., 132, pp. 346-349, 2008.

[10] M.B. Tavakoli, "Annual background radiation in the city of Isfahan," Med SciMonit, 9, pp. 7-10, 2003.

[11] E. Svoukis, H. Tsertos, "Indoor and outdoor In situ high-resolution gamma radiation measurements in urban area of Cyprus," RadiatProtDosim., 123(3), pp. 384-390, 2007.

[12] R. Rangaswamy, E. Srinivasa, M.C. Srilatha, JadiyappaSannappa, "Measurement of terrestrial gamma radiationdose and evaluation of annual effective dose inShimoga District of Karnataka State, India," Radiation Protection and Environment, Vol. 38, Issue 4, pp.154-159, 2005.

[13] Ononugbo, C.P., Avwiri, G.O. and Tutumeni, G., "Estimation of indoor and outdoor effective doses from gamma dose rates of residential building in emelogu village in rivers state, Nigeria," International Research Journal of Pure and Applied Physics, Vol.3, No.2, pp.18-27, 2015.

[14] AllawiHamedAlasadi, Azhar S. Alaboodi, Lubna A. Alasadi, Ali AbidAbojassim, "Survey of absorbed dose rates in air of Buildings Agriculture and Sciences inUniversity of Kufa at Al-Najaf Governorate, Iraq,'Journal of Chemical and Pharmaceutical Research, 8(4), pp.1388-1392, 2016.

[15] Nikl, L. B. Sztanyik, "External indoor and outdoor gamma exposures in Hungary during the period of 1983-86," Radiat. Prot. Dosim., 24 (1/4), pp. 387-389, 1988.

[16] International Commission on Radiation Units and Measurements, ICRU Report 53, "Gamma-ray spectrometry in the environment," 1994. Othman, and T. Yassine, "Natural radioactivity in the Syrian environment," Sci. of Tot. Environ, 170, pp.119-124, 1995.

[17] M. Tzortzis, H. Tsertos, S. Christofides and G. Christodoulides, "Gamma-ray measurements of naturally occurring radioactive samples from Cyprus characteristic geological rocks," Radiat. Measur, 37, pp. 221-229, 2003.

[18] X. S. Clouvas and M. Antonopoulos-Domis, "Radiological Maps of indoor and outdoor gamma dose rates in Greek Urban areas obtained by in-situ gamma spectrometry," Radiat. Prot. Dosim., 112 (2), 267-275, 2004.

[19] X. S. Clouvas and M. Antonopoulos-Domis, "Extended survey of indoor and outdoor terrestrial gamma radiation in Greek Urban areas by in-situ gamma spectrometry with portable Ge detector," Radiat. Prot. Dosim., 94 (3), pp. 233-245, 2001. B. Petalas, E. Vogiannis, D. Nikolopoulos, and C. P. Halvadakis, "Preliminary survey of outdoor gamma dose rates in Lesvos Island (Greece)," Radiat. Prot. Dosim., 113 (3), pp. 336-341, 2005.

[20] M. M. Auwal, "Determination of absorbed and effective dose from natural background radiation with portable HPGe detector," M.Sc. thesis, Ahmadu Bello University, Zaria, Nigeria, 2000.

[21] N. Karunakara, I. Yashodhara, K. Sudeep Kumara, R.M. Tripathi, S.N. Menon, S. Kadam and M.P. Chougaonkar, "Assessment of ambient gamma dose rate around a prospective uranium mining area of South India-A comparative study of dose by direct methods and soil radioactivity measurements," Results in Physics 4, pp. 20-27, 2014.

[22] S. Selvasekarapandian, K.S. Lakshmi, G.M. Brahmanandhan, V. Meenakshisundaram, "Indoor gamma dose measurement along the east coast of Tamilnadu, India using TLD," IntCongrSer, 1276, pp. 327-328, 2005.

[23] United Nation Scientific Committee on the Effects of Atomic Radiation, "Sources and Biological Effects of Ionizing Radiation," (Report to the General Assembly) New York, United Nations, 2000.

[24] United Nation Scientific Committee on the Effects of Atomic Radiation, "Sources and Biological Effects of Ionizing Radiation," (Report to the General Assembly) New York, United Nations, 2008.

[25] SadeghHazrati, Abbas NaghizadehBaghi, HadiSadeghi, Manouchehr Barak, SaharZivari, and SoheilaRahimzadeh, "Investigation of natural effective gamma dose rates case study: Ardebil Province in Iran", Iranian J Environ Health Sci Eng. v 9(1), pp 1, 2012.

[26] Gamma-scout Manual-EN, https://www.gammascout.com/, [Accessed on 10 December, 2017 ].

[27] http://en.banglapedia.org/index.php?title=File:ShahbagThana.jpg, [Accessed on 10 December, 2017]. 
[28] United Nation Scientific Committee on the Effects of Atomic Radiation, "Sources and Effects of Ionizing Radiation", (Report to the General Assembly), Annex B: Exposures from natural radiation sources, New York, United Nations,Vol. 1, 2000.

[29] BahreyniToossi MT, Bayani S, Yarahmadi M, Aghamir A, Jomehzadeh A, Parast MH, Tamjidi A. Gonad, "Bone marrow and effective dose to the population of more than 90 towns and cities of Iran, arising from environmental gamma radiation", Iran J Radiat Res., Vol 7, pp 41-47, 2009.

[30] Bouzarjomehri F, Ehrampoush M., "Gamma background radiation in Yazd province; a preliminary report", Iran J Rad Res., Vol 3, pp 17-20, 2005.

[31] UNSCEAR. REPORT - Sources and effects of ionizing radiation, Annex B: Exposures of the public and workers from various sources of radiation, New York: United Nations Scientific Committee on the effects of atomic radiation, Vol. 1, 2008.

[32] N. Sulekha Rao, KajoriParial, Hiroaki Koide, D. Sengupta, "Measurement of environmental external gamma radiation dose rate outside the dwellings of southern coastal Odisha, eastern India", Current Science, Vol 109(3), pp 600-603, 2015.

[33] ICRP 2007. The 2007 Recommendation of the International Commission on Radiological Protection. ICRP Publication 103. Ann. ICRP 37(2-4), 2007. 